\title{
Diagnosing Equipment Malfunctions Using Light Indication and Sensors \\ ${ }^{1}$ Rustem R. Ziyatdinov, ${ }^{2}$ Dmitriy A. Lobachev, ${ }^{3}$ Aleksandr A. Shabaev, ${ }^{4}$ Ramil T. Nasibullin \\ ${ }^{1-4}$ Kazan Federal University \\ Email: rust.kfu@gmail.com
}

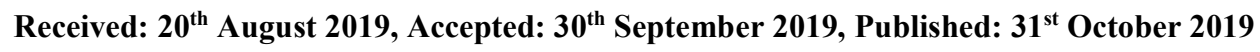

\begin{abstract}
Diagnostics of production equipment is an urgent task for modern enterprises. It allows timely identifying the emerging equipment malfunctions, prevent their occurrence and switch from emergency repairs to scheduled preventive repairs. One of the ways to identify a malfunction is to visually inspect the equipment itself, use the instrumentation readings and the light indication of equipment control systems. Moreover, the presence of a large amount of information causes difficulties when the maintenance personnel conducts diagnostics and requires high qualifications. To solve this problem, one can use the image recognition and the formation of recommendations for maintenance and repair using an intelligent system. Image recognition is one of the most sought-after functions of modern control systems used in various activity fields.

This article proposes a malfunction diagnosing method by light indication, based on the use of artificial intelligence elements. The input information is represented by the images of the status of light indicators of the equipment and the instrumentation readings. The resulting image is pre-processed and classified in order to determine the equipment condition. The correlation of the light indication image to a certain state of equipment is carried out using one of the classification algorithms. The most promising classification methods in image recognition problems can be considered the methods of support vectors and $\mathrm{k}$ - the nearest neighbors. Further, on the basis of the received equipment condition, recommendations for its maintenance and repair are formed with the help of an expert system. To develop an expert system, we used a production model that allows applying simple and accurate mechanisms for using knowledge.

Thus, an intelligent system will allow diagnosing production equipment faster and more efficiently, without imposing high requirements on the staff qualifications.
\end{abstract}

\section{Keywords}

Equipment Diagnostics, Expert Equipment Diagnosing System.

\section{Introduction}

Increased requirements for the quality of products require continuous improvement of production technology. One way to solve this problem is the integrated enterprise automation. At the same time, it is important to carry out timely equipment diagnostics for the transition from emergency repairs to scheduled preventive repairs [1,2]. At the same time, the variety of used production equipment and automation means creates additional difficulties in integrating all the existing subsystems into a single system.

One of the possible directions for solving such problems is the use of intelligent systems with the elements of technical vision. Previously, the development of such systems was constrained by high demands on computing resources. The emergence of new image processing and classification techniques, increased productivity of modern computing systems removes almost all the problems of system implementation based on technical vision. Image recognition problems are encountered in a wide variety of fields: from fault detection in diagnosing systems to driving unmanned vehicles [3].

Management of production equipment is carried out using industrial controllers. Information at the inputs and outputs of industrial controllers determines equipment condition. Therefore, to diagnose the condition and malfunctions of industrial equipment, one can use the light indication on the input/output modules of industrial controllers. At the same time, the light indication on the industrial controller can have hundreds of indicators, and when maintenance personnel detects and diagnoses a malfunction, difficulties arise in the data interpretation. In addition, some malfunctions may require in-depth theoretical knowledge and extensive practical experience.

Expert systems can be used to solve the problem of diagnosing equipment malfunctions [4, 5]. The task of the expert system in this case is to identify equipment malfunctions according to the input data and issue of recommendations for maintenance or repair, if necessary.

\section{Methods}

To solve the problems of image recognition, it is necessary to use one of the image classification methods. The classification task is, in general terms, a formalized task where there are many objects divided into classes in a certain way. For given objects, it is known which classes they belong to. The class affiliation of all other objects is unknown. The classification task is to determine the class membership (classify) of an arbitrary object.

To date, there are many methods for classifying images that differ in quality and recognition speed. In the work [6], a comparative analysis of the following classification algorithms was carried out: the support vector method, the EM algorithm, the method $\mathrm{k}$ - the nearest neighbors, the decision tree and the naive Bayesian classifier. The work results showed that the most promising classification methods in image recognition tasks are the support vector methods and $\mathrm{k}$ - 
the nearest neighbors. The fastest learning time has the method $\mathrm{k}$ - the nearest neighbors, but it is inferior to the support vector method, both in the recognition time of the test sample and in the number of correctly recognized images. In this problem, the recognition speed is not a critical parameter, therefore, one can use both the method $\mathrm{k}$ - the nearest neighbors, and the support vector method.

Most often, classical neural networks (a network of radial basis functions, a multilayer perceptron, etc.) are used in image recognition tasks. However, in some cases, the use of classical neural network architectures may have little effectiveness for the following reasons:

- recognized images often have large sizes, which leads to an increase in the structure of the neural network;

- a wide variety of parameters increases the system size, which leads to an increase in the need for a larger training set and, consequently, an increase in the complexity of calculations and the time required for the system training;

- to ensure the high efficiency of the recognition system, the use of several neural networks, trained with different order of providing the initial images and the initial values of synaptic coefficients, is required. This leads to an increase in the complexity of solving the problem and its execution time;

- high sensitivity to various changes in the geometry of the input images, such as a change in the shooting angle, image scale and other distortions [7-9].

However, classical neural networks are devoid of the above disadvantages to solve this problem.

A production model was used to develop an expert system. Production models are the most common models for the current day in which the knowledge is represented using rules of the form: If - then [10]. When using such models, knowledge-based systems have the ability to:

- use a simple and accurate mechanism for using knowledge;

- represent knowledge with high uniformity, described by a single syntax.

\section{Results and Discussion}

Let us consider the diagnosing technique for the equipment condition using the example of the HURTH ZK-10 gearchamfering machine. This machine is controlled using an industrial controller with discrete input-output modules. The input and output data of the controller determine the machine condition. To diagnose the condition, 59 discrete input signals and 39 output signals are used (Figure 1). The installer or service engineer determines the equipment condition and identifies malfunctions, if any, under this indication.

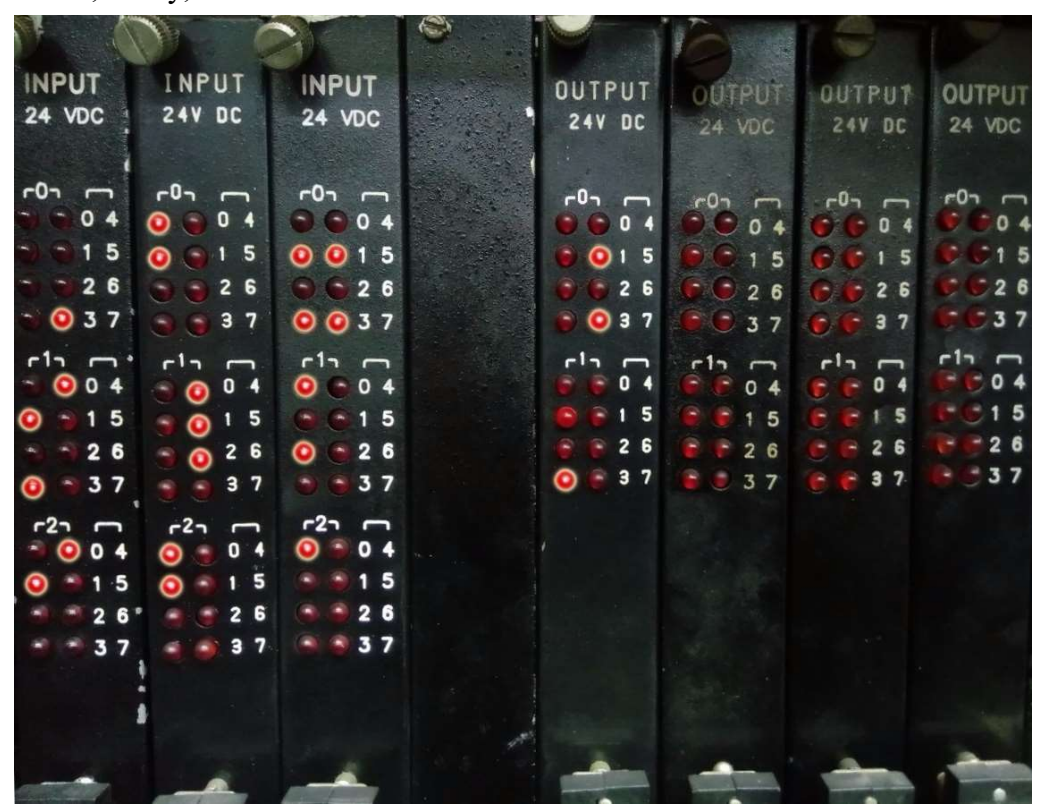

Figure 1: Machine Condition Alarm

For the automated diagnostics of machine condition, it is required to obtain data from the managing industrial controller. However, direct connection to this controller is difficult and requires additional financial costs. Therefore, the state of input and output signals can be determined by the light indication used on the input/output modules using technical vision elements. One can get the image of the equipment indicator lights in various ways, for example, using a smartphone camera or a stationary camera. Then it is necessary to carry out preliminary image processing and classification of the resulting image. Further, using the expert system, they determine the equipment condition and offer recommendations for maintenance or repair.

Pre-processing is needed to identify luminous indicators against the background of the rest of the image, for example, using color filtering. The result of preliminary processing of the original image is shown in Figure 2. 

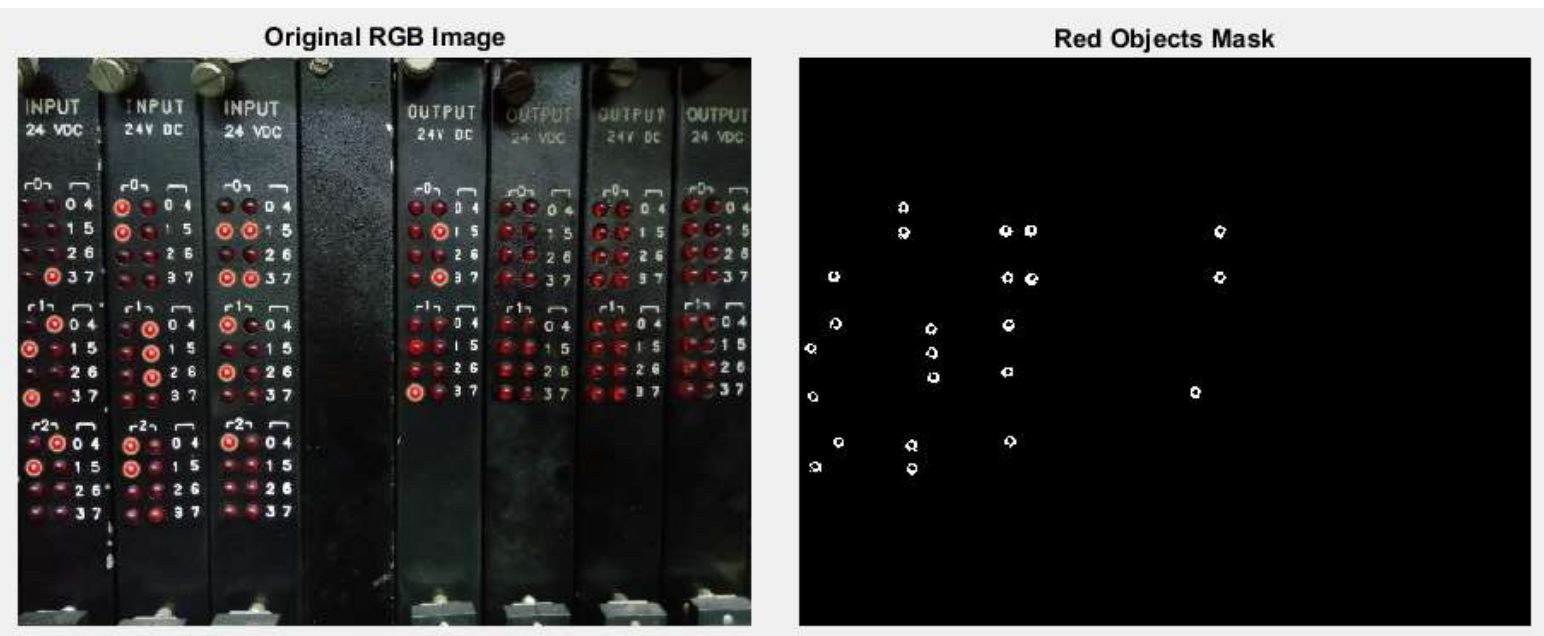

Figure 2: The Result of Preliminary Processing of the Original Image

Then, one need to convert the resulting image into the view necessary for the neural network operation. To this end, the image can be converted to binary matrix format. To do this, the image is divided into cells. The number of cells is determined by the size and number of light indicators. The cell that contains the luminous indicator is indicated as 1 , the remaining cells - as 0 . An example of a binary matrix formation is shown in Figure 3.

\begin{tabular}{|l|l|l|l|l|l|l|l|l|l|}
\hline 0 & 0 & $1 *$ & $1 *$ & 0 & 0 & 0 & $1 *$ & 0 & 0 \\
\hline 0 & 0 & $1 *$ & 0 & 0 & 0 & 0 & 0 & $1 *$ & $1 *$ \\
\hline 0 & 0 & 0 & 0 & 0 & 0 & 0 & 0 & $1 *$ & 0 \\
\hline 0 & $1 *$ & $1 *$ & 0 & 0 & 0 & $1 *$ & 0 & $1 *$ & 0 \\
\hline 0 & $1 *$ & 0 & 0 & $1 *$ & 0 & 0 & 0 & 0 & 0 \\
\hline 0 & 0 & 0 & 0 & 0 & 0 & 0 & $1 *$ & $1 *$ & 0 \\
\hline $1 *$ & 0 & 0 & 0 & 0 & 0 & 0 & $1 *$ & 0 & 0 \\
\hline 0 & 0 & $1 *$ & 0 & 0 & $1 *$ & 0 & 0 & 0 & 0 \\
\hline
\end{tabular}

Figure 3: Image Representation as a Binary Matrix

To diagnose a malfunction, a neural network trained on reference images, which are also presented in matrix form, is used.

The structural diagram of the diagnostic system will have the following form (Figure 4).

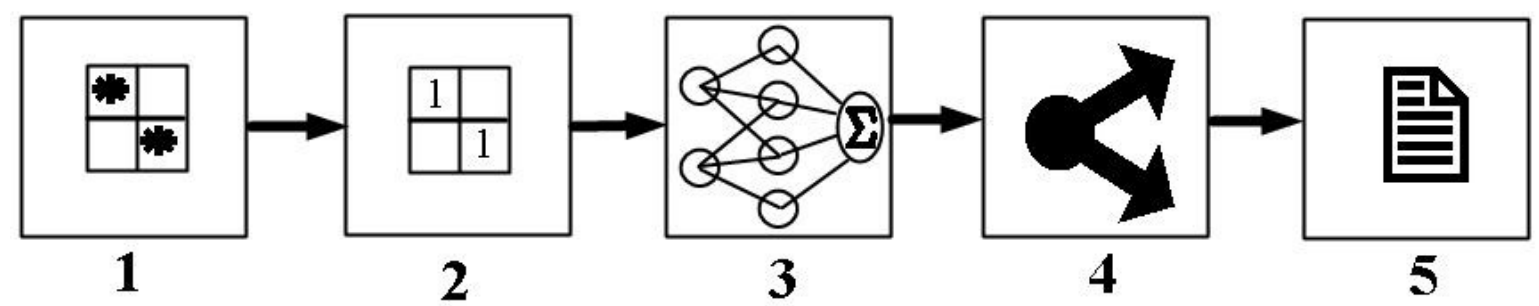

Figure 4: Block Diagram of the Diagnostic System

The initial data, presented in the form of indicators, first enter the image formation unit (1). In this unit, the original image is formed using the camera. Then, the condition image of the light indication is fed to a unit intended for conversion to a recognition system format (2). In this unit, the image is pre-processed and converted into a binary matrix format. A pretrained unit of the neural network (3) identifies the features of the original image and makes a decision on whether the initial image belongs to any typical equipment condition. The conclusion about belonging of the original image of any 
equipment malfunction is determined using the block (4). Then, the expert system (5), based on the knowledge base, generates recommendations for the maintenance or repair of diagnosed equipment.

A rule base containing 63 rules has been developed for this machine. Examples of received rules:

1. If $\mathrm{I} 000=1$ and $\mathrm{I} 001=1$ then "Check the loading/unloading buttons of a part".

2. If I002 = 1 and I003 = 1 then "Check the input/output buttons of the milling slides".

3. If $\mathrm{I} 006=1$ and $\mathrm{I} 007=1$ then "Check the automatic/commissioning switch."

4. If $\mathrm{I} 012=1$ and $\mathrm{I} 013=1$ then "Check the cutter motor speed switch".

5. If $\mathrm{I} 014=1$ and $\mathrm{I} 015=1$ then "Check the engine speed switch and high speed division switch".

6. If I102 = 1 and I103 = 1 then "Check the contacts of KM3 and KM4 starters".

7. If I104 = 1 and I105 = 1 then "Check the contacts of KM5 and KM6 starters".

8. If $\mathrm{I} 107=1$ and $\mathrm{I} 110=1$ then "Check the contacts of KM7 and KM8 starters".

9. If $\mathrm{I} 112=1$ and I113 = 1 then "Check the contacts of KM11 and KM12 starters".

...

60. If I021 = 1 and I022 = 1 then "Check the cutter motor reverse switch".

61. If Q700 = 1 and Q701 = 1 then "Check the condition of the switch and reverse actuator of the cutter motor or output module $700 "$.

62. If $\mathrm{I} 101=1$ and $\mathrm{Q} 701=1$ then "Check the condition of KM 4 contacts; input module 100 and output module 700".

63. If $\mathrm{I} 102=1$ and $\mathrm{Q} 700=1$ then "Check the condition of KM 3 contacts, input module 100 and output module 700".

\section{Summary}

Thus, the algorithm of the intelligent diagnosing system will contain the following steps:

1. Formation of the original graphic image intended for recognition.

2. Pre-processing of the original image.

3. Encoding a graphic image into a binary matrix.

4. Isolation of qualitative characteristic features and decision-making on whether the original image belongs to any typical malfunction.

5. Formation of recommendations for the maintenance or repair of equipment.

\section{Conclusions}

The system allows determining the status of production equipment and issue recommendations for its repair based on the input and output signals of its control system. At the same time, the speed of the equipment diagnosing process increases many times over in comparison with the time spent on diagnosing malfunctions by maintenance personnel. This system can be easily scaled and adapted to a wide range of different equipment. Another feature of the system use is that the diagnosing process itself can be performed by low-skilled service personnel. All this allows reducing equipment downtime due to malfunctions and increase production efficiency

\section{Acknowledgements}

The work is performed according to the Russian Government Program of Competitive Growth of Kazan Federal University.

\section{References}

[1] Ziyatdinov R.R., Mukhametzyanov V.R. and Nabiullina G.I. Application of neural networks for the diagnosis of depth sucker rod pumps // International Journal of Applied Engineering Research, Volume 10, Issue 24, 1 December 2015, Pages 45022-26.

[2] Sharafieva L.N., Ziyatdinov R.R. Application of artificial neural network in the problems of diagnosis of borehole pumps / Information Technology. Automation. Actualization and solving the problems of training highly qualified personnel: Proceedings of the international conference - Naberezhnye Chelny, 2016. - p.83-87. (in Russian)

[3] Ziyatdinov R., Shigabiev R. and Talipov D. Automated road marking recognition system. IOP Conf. Series: Materials Science and Engineering 240 (2017) 012070 doi:10.1088/1757-899X/240/1/012070.

[4] Expert systems [Electronic resource]. - Access mode: http:/www.aiportal.ru/articles/expert-systems/expertsystems.html. (access date 22.05.2019).

[5] CAD expert systems: Textbook / A.L. Ezdakov. — M. : ID "FORUM": INFRA-M, 2019. — 160 p.

[6] Ziyatdinov R R, Biktimirov R A and Klochkova K V. The comparative analysis of classification of algorithms in the problems of pattern recognition. Journal of Fundamental and Applied Sciences, [S.1.], v. 9, n. 1S, p. 1790-97, feb. 2017. ISSN 1112-9867.

[7] Yu N., Notkin B.S., Sedov V.A. Neuroiterative algorithm of tomographic reconstruction of the distributed physical fields in the fibreoptic measuring systems // Computer optics. - 2009. - V. 33. -№ 4. - P. 446-455. 
[8] Automated road sign recognition system for ADAS systems / Ziyatdinov R.R., Biktimirov R.A. // In the collection: Innovative Engineering Technologies, Equipment and Materials - 2017 (MNTK "IMTOM-2017"), Materials of the VIII International Scientific and Technical Conference. 2017. P. 153-158.

[9] Wilson D.R., Martinez T.R. The general inefficiency of batch training for gradient descent learning // Neural Networks. - 2004. -V. 16. - № 4. - P. 1429-1451.

[10] Production knowledge model. [Electronic resource]. - Access mode: http://www.aiportal.ru/articles/knowledgemodels/production-model.html. (access date 22.05.2019). 